\title{
The future of personalized care: humanism meets artificial intelligence
}

\author{
Darshana Shah, PhD ${ }^{1}$
}

\section{KEYWORDS}

Author affiliations are listed at the end of this article.

Correspondence to: Darshana Shah, PhD Marshall University Joan C. Edwards School of Medicine shah@marshall.edu

artificial intelligence, humanism

Another exciting year for the Marshall Journal of Medicine has passed, giving us the opportunity to both reflect on the accomplishments of the past year and summarize recent developments. The readership distribution of the Marshall Journal of Medicine spans the globe, including over 169 countries and 32,000 plus downloads. We continue to expand our scope by incorporating new submission categories such as "At the 'Heart' of Healthcare" and "Machine Learning in Rural Health." This issue particularly propels us forward with a focus on the humanism of health care.

Dr. Lakhani's reflection is a poignant reminder of the impact we have on others as human beings, society members, and health-care providers. His portrait of perseverance, determination, and care is an inspirational guide for readers to contemplate the privileges and responsibilities that accompany mentorship and our role as health care providers, as well as human beings.

Ms. White's editorial forces us to focus on "the human factor" of healthcare, or the belief that every person throughout the healthcare system — caregiver and patient alike-is first and foremost a human being. She analyzes the importance of health literacy ${ }^{2}$ and the positive outcomes that arise from a system that embraces the complete care of individuals and communities. Her contemplation of a fractured system, and her solution to strengthen it, provide us with a new, sustainable vision of health care for the future.

We are on the edge of a digital revolution that will drive healthcare quality improvement. Artificial intelligence holds tremendous promise for transforming the provision of health care services in resource-poor settings ${ }^{3}$. Dr. Khitan and his colleagues shed light on how machine learning could be utilized to identify patients with end-stage renal disease (ESRD) at higher risk for complications and thereby, improve patient outcomes. Such Al tools can empower the healthcare providers and patients alike with capabilities that enable more humanistic care in today's electronic health record world.

Incorporating tenets of humanism into health care, where everyone is treated with dignity and 
respect, will foster a more inclusive culture and subsequently stronger relationships with patients and communities. This approach can ultimately result in the provision of care that is compassionate and high quality while providing diverse values, beliefs, behaviors, and delivery of care that meet the patient's social and cultural needs.

\section{AUTHOR AFFILIATIONS}

1. Marshall University Joan C. Edwards School of Medicine, Huntington, West Virginia

\section{REFERENCES}

1. Lee Roze des Ordons A, de Groot JM, Rosenal T, et al. Perspect Med Educ. 2018;7:318. https://doi. org/10.1007/s40037-018-0455-4

2. Hudson S, Rikard RV. 2018. The case for health literacy: Moving from equality to liberation. NAM Perspectives. Commentary, National Academy of Medicine, Washington, DC. https://doi. org/10.31478/201805a

3. Wahl B, Cossy-Gantner A, Germann S, et al. Artificial intelligence (Al) and global health: how can Al contribute to health in resource-poor settings? BMJ Global Health. 2018;3:e000798. 